\title{
The impact of cooling methods on the maximum temperature of the processed object during side milling
}

\author{
Lukasz Nowakowski ${ }^{1, *}$, Michat Skrzyniarz ${ }^{1}$, and Edward Miko ${ }^{1}$ \\ ${ }^{1}$ Kielce University of Technology, Department of Manufacturing Engineering and Metrology, Aleja Tysiąclecia Państwa Polskiego 7; \\ 25-314 Kielce; Poland
}

\begin{abstract}
This article presents the changes in temperature distribution concerning the processed object during side milling. The analysis included the influence of selected cooling methods (cold air from vortex tube, cooling with compressed air, and without cooling) on the maximum temperature of the processed object during the process of milling. Measurement of the maximum temperature was conducted with the use of thermocouples and a thermal camera.
\end{abstract}

\section{Introduction}

During the process of machining, heat is generated as a result of elastic and plastic deformation of the material and of friction occurring between the contact surface and the processed material[1-8]. An additional source of providing heat to the area of machining it the work of friction of chips against the surface of the tool. The temperature present within the area of machining influences the shape and size accuracy of created objects [9-14]. It has a significant meaning during performance of precision parts of machines with high reliability[1523], as well as prototypes [24] i.e. contactless face seals [4-8] of gyroscopes etc. [24-26]. It may, additionally, cause deformation of the processed object and the tools [27-35]. Excessive amount of heat delivered to the tool results in a loss of cutting properties of the tools and, consequently, in damaging the tool, which has a significant impact on production costs and efficiency of performed processing. Monitoring of temperature is very important due to the need for selection of proper conditions to perform the process of machining. Heat is dissipated from the machining area through the tool, processed object, created chips, as well as other sources, e.g. by lifting or usage of liquids: cooling liquids and cooling and lubricating liquids. The largest amount of heat is dissipated from the machining area through chips. That is why its shape, cross-section, and method of tearing off is one of the essential factors that influence distribution of temperatures within the area of machining. Conducting the process of machining with the use of cutting fluid cools the tool and the processed object. Additionally, it decreases friction that occurs within the area of machining, which leads to a lower amount of released heat and lower demand for power required to conduct processing [36-38].

The measurement of average temperatures within the machining area has been mainly based on contact methods by using thermocouples, as well as contactless methods that are based on the phenomenon of heat radiation [39].

\section{Methods}

Tests were performed on a vertical machining center AVIA VMC 800. The tool used for the experiment was a tow-edge solid end mill produced by Sandvik Coromant - R216.32-12025-AK38A H10F with diameter of 12 $\mathrm{mm}$. Table no. 1 presents the basic geometrical dimensions of the tool used for processing.

Table 1. Geometrical dimensions of the tool.

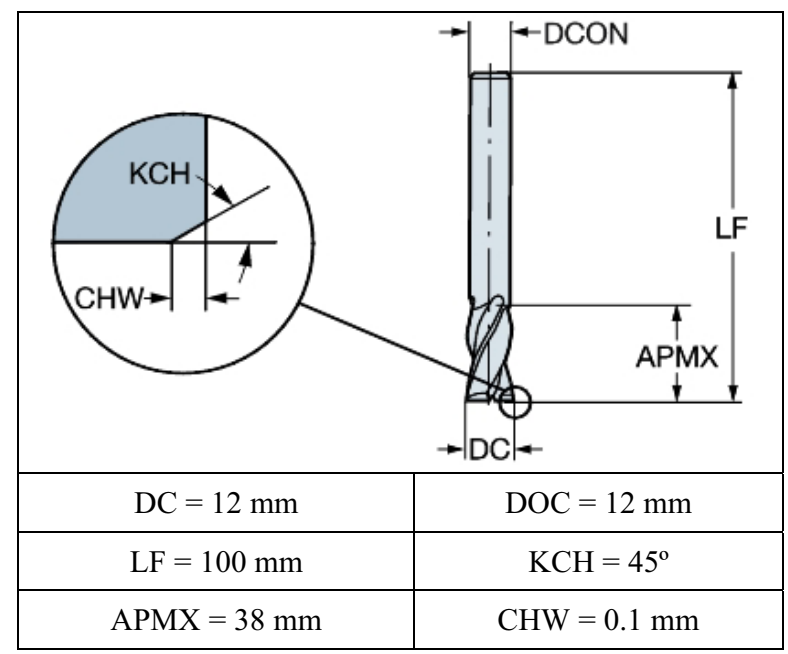

The process of side milling was performed at depth of $\mathrm{a}_{\mathrm{p}}=30 \mathrm{~mm}$, side feed $\mathrm{a}_{\mathrm{e}}=0.5 \mathrm{~mm}$ and feed rate of $\mathrm{f}=300 \mathrm{~mm} / \mathrm{min}$. Turning speed of the spindle was $10,000 \mathrm{rpm}$. The tested material was a $30 \times 40 \times 10 \mathrm{~mm}$ rectangular sample made of copper. Figure no. 1 presents geometrical dimensions of the sample along with the arrangement of thermocouples. K-type thermocouples of

Corresponding author: skrzyniarzmichal@gmail.com 
$1 \mathrm{~mm}$ in diameter were used to measure the temperature. They were placed $10 \mathrm{~mm}$ inside the processed object.

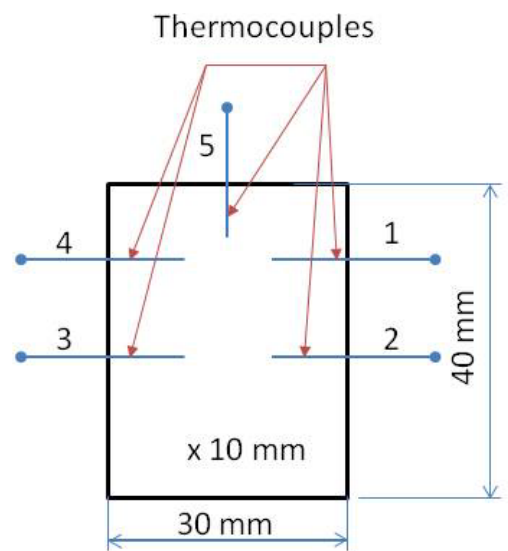

Fig. 1. Geometrical dimensions of the sample.

Apart from contact measurement conducted with the use of thermocouples, the experiment station also included a thermal camera Flir i7. Specification of that camera has been presented in table no. 2 .

Table 2. Specification Flir i7 camera

\begin{tabular}{|c|c|}
\hline Resolution & $\mathbf{1 4 0 x 1 4 0}$ pixels \\
\hline Thermal Sensitivity & $0.1^{\circ} \mathrm{C}$ \\
\hline Temperature Range & $-20 \div 250^{\circ} \mathrm{C}$ \\
\hline Accuracy & $\pm 2^{\circ} \mathrm{C}$ \\
\hline Spectrum & $7.5 \div 13 \mu \mathrm{m}$ \\
\hline Frame Rate & $9 \mathrm{kHz}$ \\
\hline Emissivity & $0.1 \div 1.0$ \\
\hline
\end{tabular}

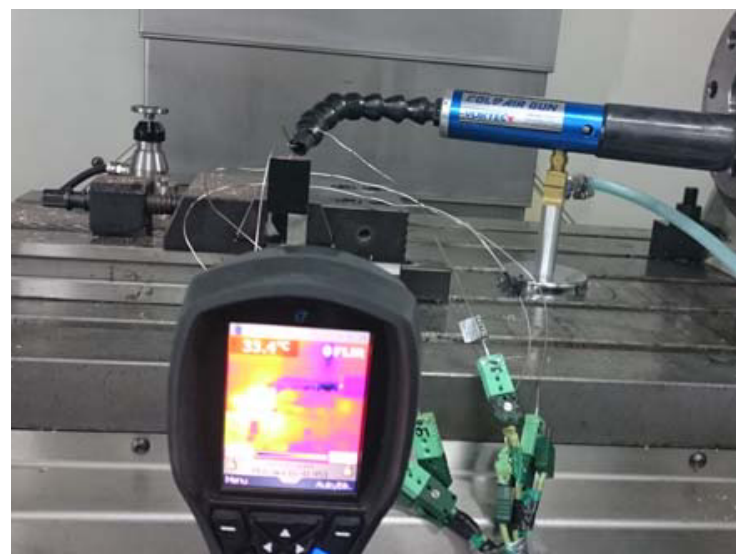

Fig. 2. Experiment station used for measurement.

The experiment was to conduct machining attempts and measure the average and maximum temperatures inside the processed object and on its front wall. The machining processed was conducted several times on one sample in order to determine the distribution of temperatures together with changes in the distance of the measurement point from the source of heat. Additionally, it was decided to conduct an experiment without the use of cooling, with the use of cooling for the machining area by using compressed air, and by using a vortex tube. That device allows division of the stream of compressed air into a stream of hot and cold air that is directed at the machining area. Figure no. 2 presents the experiment station used for measurements.

\section{Results}

Table no. 3 presents the average and maximum temperature values obtained during processing without cooling. It was observed that the highest values of average temperatures were noted at the distance of $2 \mathrm{~mm}$ of thermocouples from the machining area. The farther the source of heat from thermocouples, the lower the temperature. The analogous dependency has been noted for measurement made by the thermal camera.

Table 3. Temperature values obtained Turing process without cooling.

\begin{tabular}{|l|c|c|c|c|c|c|}
\hline \multirow{2}{*}{ Temp, ${ }^{\circ} \mathbf{C}$} & \multicolumn{6}{|}{ Distance of thermocouples from the } \\
machining area, mm \\
\cline { 2 - 7 } & $\mathbf{4 . 5}$ & $\mathbf{4}$ & $\mathbf{3 . 5}$ & $\mathbf{3}$ & $\mathbf{2 . 5}$ & $\mathbf{2}$ \\
\hline Thermo. 1 & 101 & 108 & 108 & 128 & 134 & 140 \\
\hline Thermo. 2 & 90 & 98 & 108 & 114 & 119 & 123 \\
\hline Thermo. 3 & 75 & 80 & 88 & 93 & 95 & 98 \\
\hline Therm. 4 & 82 & 87 & 96 & 103 & 105 & 110 \\
\hline Thermo. 5 & 90 & 97 & 108 & 115 & 119 & 124 \\
\hline Camera & 97 & 104 & 115 & 124 & 131 & 142 \\
\hline
\end{tabular}

Figure 3 presents charts that describe the change in temperatures during the processing without cooling at the distance of thermocouple distribution of $2.5 \mathrm{~mm}$ from the source of heat in form of the operating tool. It was noticed that the maximum temperature of $134^{\circ} \mathrm{C}$ was obtained for thermocouple no. 1 that was the farthest from the source of heat. Accumulation within that area is caused by the fast and constant flow of heat from the beginning of the processing. Average results of temperature measurement for thermocouples 1 and 2 differ from one another although they have been placed on the same wall. It is caused by the distance of thermocouples from the upper and lower edge where some part of the heating energy directed to thermocouple no. 2 is obtained by the grip, as well as the lower unprocessed part of the sample. Analogous situations occur in the case of other measurements.

Table no. 4 contains results of measurement of the average and maximum temperatures for processing with the same technological parameters. Additionally in that case, cooling of the machining area with a vortex tube was applied. During measurement, the noted temperature of air at the outlet from the tube was about $-29^{\circ} \mathrm{C}$. After analyzing the results, it was noticed that cooling of the machining area with cold air does not significantly affect the results of the average temperatures noted by the vortex tube and the maximum temperature recorded with the thermal camera. 


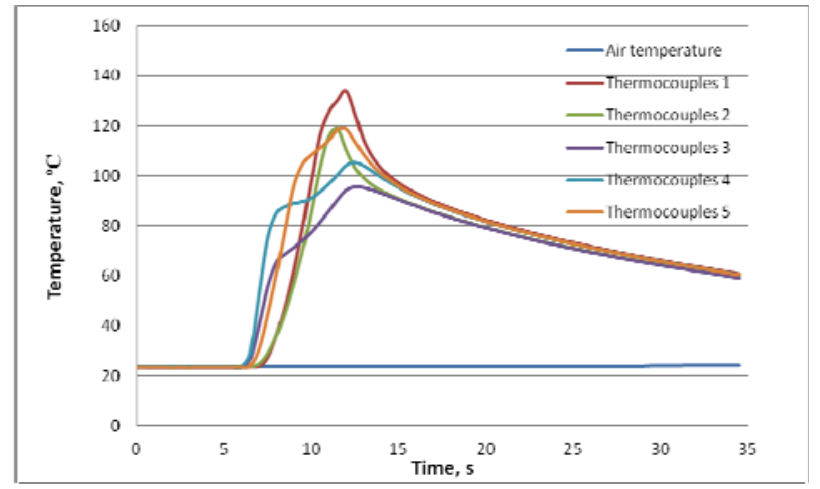

Fig. 3. Change the temperatures during the processing without cooling at the distance of thermocouple distribution of $2.5 \mathrm{~mm}$.

Table 4. Temperature values obtained Turing process with cooling by Vortex tube.

\begin{tabular}{|c|c|c|c|c|c|c|}
\hline \multirow{2}{*}{ Temp, ${ }^{\circ} \mathrm{C}$} & \multicolumn{6}{|c|}{$\begin{array}{l}\text { Distance of thermocouples from the } \\
\text { machining area, } \mathrm{mm}\end{array}$} \\
\hline & 4.5 & 4 & 3.5 & 3 & 2.5 & 2 \\
\hline Thermo. 1 & 109 & 117 & 126 & 128 & 147 & 154 \\
\hline Thermo. 2 & 100 & 104 & 112 & 114 & 120 & 126 \\
\hline Thermo. 3 & 83 & 84 & 90 & 91 & 95 & 98 \\
\hline Thermo. 4 & 89 & 93 & 99 & 100 & 105 & 110 \\
\hline Thermo. 5 & 98 & 105 & 110 & 112 & 118 & 124 \\
\hline Camera & 99 & 115 & 120 & 120 & 126 & 142 \\
\hline
\end{tabular}

The maximum average temperature for processing with cooling is by $14^{\circ} \mathrm{C}$ higher than in the case of processing without cooling. It may be caused by lack of dissipation of heat from machining area through the tool that heated to a high temperature due to conducting the previous processing.

Table 5. Temperature values obtained Turing process with cooling by compressed air.

\begin{tabular}{|l|c|c|c|c|c|c|}
\hline \multirow{2}{*}{ Temp, } & \multicolumn{6}{|}{ Distance of thermocouples from the } \\
& \multicolumn{2}{|c|}{ machining area, mm } \\
\cline { 2 - 8 } & $\mathbf{4 . 5}$ & $\mathbf{4}$ & $\mathbf{3 . 5}$ & $\mathbf{3}$ & $\mathbf{2 . 5}$ & $\mathbf{2}$ \\
\hline Thermo. 1 & 108 & 113 & 125 & 131 & 137 & 146 \\
\hline Thermo. 2 & 96 & 99 & 109 & 114 & 119 & 126 \\
\hline Thermo. 3 & 81 & 84 & 89 & 86 & 97 & 101 \\
\hline Thermo. 4 & 88 & 91 & 100 & 104 & 108 & 113 \\
\hline Thermo. 5 & 97 & 100 & 110 & 115 & 120 & 127 \\
\hline Camera & 97 & 101 & 118 & 120 & 125 & 138 \\
\hline
\end{tabular}

In the case of processing with cooling in the form of compressed air, values of temperatures were recorded in table no. 5. The recorded air temperature at the nozzle outlet was $24^{\circ} \mathrm{C}$. The average and maximum recorded temperatures in that case of processing do not significantly deviate from the values recorded for previous processing attempts with cooling by the use of compressed air from a vortex tube or without cooling.

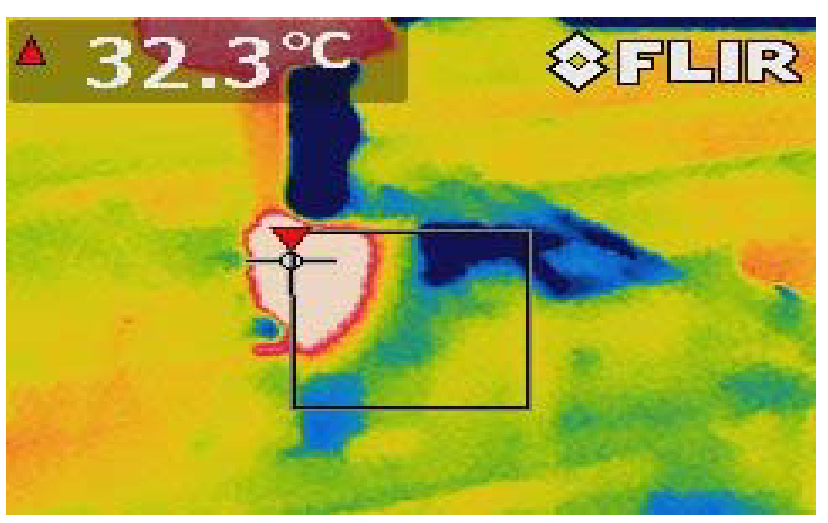

Fig. 4. Example image obtained with the thermal camera during measurement.

Figure no. 4 presents the example image obtained with the thermal camera during measurement. The noted temperature in that case was $32.3^{\circ} \mathrm{C}$ and was observed during the start of machining. In the picture, one may clearly notice the direction of release of heat to the remaining part of the sample, while the temporary maximum temperature was in places where the material was divided.

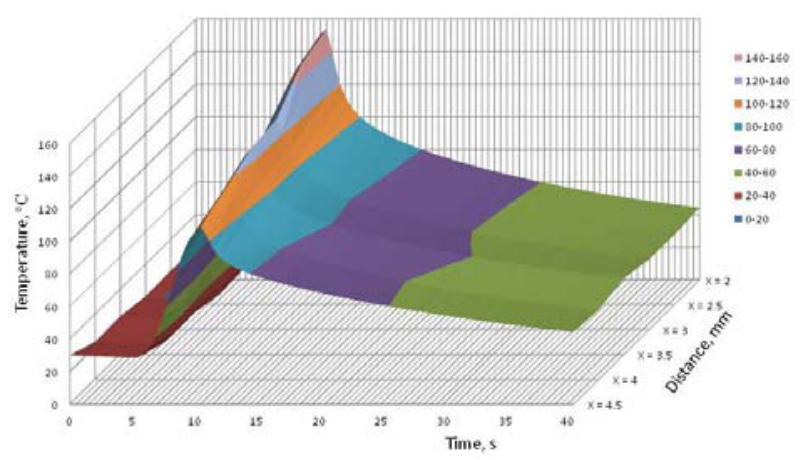

Fig. 5. Changes of the temperature during the processing with cooling by the use of a vortex tube.

Figure 5 presents the chart describing changes of temperature during the processing with cooling by the use of a vortex tube. The highest temperature was noted for that type of machining and amounted $154{ }^{\circ} \mathrm{C}$, while the cooling process of the processed object was the fastest and amounted 51 seconds to reach temperature of the processed object below $30^{\circ} \mathrm{C}$. Application of cooling in the form of compressed air required 77 seconds after finishing the process to decrease temperature of the processed object below $30{ }^{\circ} \mathrm{C}$, while in the case of processing without cooling - 104 seconds.

\section{Conclusions}

The analysis of the experiment allowed formulation of the following conclusions:

1. Processing by using the cooling method with compressed air or cold air from a vortex tube does not 
decrease the maximum average value of temperatures of the processed object.

2. The highest temperature was noted for thermocouple no. 1, which results from accumulation of heat and no dissipation of it from the area by contact with the machining grip.

3. Analysis of temperatures inside the sample performed with thermocouples is similar to values of temperatures recorded by the thermal camera.

4. The cooling method of the machining process significantly affects dissipation of heat from the processed object after the machining process is finished. 5. Maximum temperatures recorded during processing with cold compressed air result from limited heat capacity of the tool arising from accumulation of heat stored from the previous processing.

\section{References}

1. S. Hożejowska, R.Kaniowski, M. E. Poniewski, Exper. Thermal and Fluid Scienc 78, 18 (2016)

2. S. Hożejowska, R.Kaniowski, M. E. Poniewski, Inter. J. of Num. Meth. for Heat \& Fluid Flow 24, (4), 811 (2014)

3. R. Pastuszko, R. Kaniowski, EPJ Web of Conferences (Proc. Int. Conf. Experimental Fluid Mechanics 2010) 25, 02019 (2012)

4. S. Blasiak, J. E. Takosoglu, P. A. Laski, J. Therm. Sci. Techn. 9, 2 (2014)

5. S. Blasiak, P. A. Laski, J. E. Takosoglu, Int. J. Heat Mass Tran. 57, 1 (2013)

6. S. Blasiak, C. Kundera, Procedia Eng. 39, 315 (2012)

7. S. Blasiak, A. Pawinska. Int J Heat Mass Transf. 90, 710 (2015)

8. S. Blasiak, C. Kundera, Bochnia J. Procedia Eng. 39, 366 (2012)

9. L. Nowakowski, E. Miko, M. Skrzyniarz, Edited by Zolotarev I., Radolf V. Proceedings of 22nd International Conference on Engineering Mechanics 2016 (Academy of Sciences of the Czech Republic, 2016)

10. L. Nowakowski, M. Wijas, Edited by Zolotarev I., Radolf V. Proceedings of 22nd International Conference on Engineering Mechanics 2016 (Academy of Sciences of the Czech Republic, 2016)

11. L. Nowakowski, M. Miesikowska, M. Blasiak, Edited by Zolotarev I., Radolf V. Proceedings of 22nd International Conference on Engineering Mechanics 2016 (Academy of Sciences of the Czech Republic, 2016)

12. E. Miko, L. Nowakowski, Procedia Eng. 39 (2012a)

13. E. Miko, L. Nowakowski, Procedia Eng. 39 (2012b)

14. S. Adamczak, E. Miko, F. Cus, Strojniski VestnikJour. of Mech. Eng. 55,(1),45 (2009)

15. J.E. Takosoglu, Edited by Zolotarev I., Radolf V. Proceedings of 22nd International Conference on Engineering Mechanics 2016 (Academy of Sciences of the Czech Republic, 2016)

16. J.E. Takosoglu, P.A. Laski, S. Blasiak, G. Bracha, D. Pietrala. Meas. Cont. 49, 2, 2016
17. P.A. Laski, J.E. Takosoglu, S. Blasiak, Rob Auton Syst.72, 59 (2015)

18. G.F. Bracha. Edited by Zolotarev I., Radolf V. Proceedings of 22nd International Conference on Engineering Mechanics 2016 (Academy of Sciences of the Czech Republic, 2016)

19. J.E. Takosoglu, P.A. Laski, S. Blasiak, Edited by Fuis V. Proceedings of 20th International Conference on Engineering Mechanics 2014 (Brno University of Technology, Czech Republic, 2014)

20. J.E. Takosoglu, P.A. Laski, S. Blasiak. P. I. Mech. Eng. I-J. Sys. 226, 10 (2012)

21.P.A. Laski, Edited by Zolotarev I., Radolf V. Proceedings of 22nd International Conference on Engineering Mechanics 2016 (Academy of Sciences of the Czech Republic, 2016)

22. D.S. Pietrala, Edited by Zolotarev I., Radolf V. Proceedings of 22nd International Conference on Engineering Mechanics 2016 (Academy of Sciences of the Czech Republic, 2016)

23. S. Adamczak, B. Kaczmarska, J. Bochnia, Metrology and Measurement Systems, 22 (2015)

24. I. Krzysztofik, Z. Koruba J. of App. Math., Vol. 2014, 11 (2014),

25. I. Krzysztofik, Edited by Zolotarev I., Radolf V. Proceedings of 22nd International Conference on Engineering Mechanics 2016 (Academy of Sciences of the Czech Republic, 2016)

26.Z. Koruba, I. Krzysztofik, P. I. Mech. Eng. K-J. Multi-Body Dyn. 227, K1, 2013

27. J. Kumaszewski. I Zagórski, Management and Production Engineering Review, 4 (2013)

28. M. Graba, J. of Theor. And Appl. Mech. 50 (1) 23 (2012)

29. M. Graba, J. of Theor. And Appl. Mech. 51 (2) 349 (2013)

30. W. Depczyński, R. Kazała, K. Ludwinek, K. Jedynak, Mat. 9 (7), 567, 1 (2016)

31. W. Depczyński METAL 2014 23rd Inter. Conf. on Metal. and Mat. 1219 (2014)

32. S. Spadło, P. Młynarczyk, W. Depczyński Proceedings of 24th Inter. Conf. on Metall. and Mat. METAL 2015863 (2015)

33. J. Zwierzchowski, Edited by Zolotarev I., Radolf V. Proceedings of 22nd International Conference on Engineering Mechanics 2016 (Academy of Sciences of the Czech Republic, 2016)

34. D. Janecki, L. Cedro, J. Zwierzchowski, Metrology Meas. Sys. 22, 2, 2015

35. D. Janecki, J. Zwierzchowski, L. Cedro, B. Pol. Acad. Sci-Tech. Sci. 63, 3, 2015

36. M. Bartoszuk, W. Grzesik, Edited by: Outeiro, Modelling Of Machining Operations Advan. Mat. Res. 223, 231 (2011)

37. W. Grzesik,; M. Bartoszuk, P. Nieslony, Machining Science And Technology , 9, (4), 529 (2005)

38. W. Grzesik,; P. Nieslony, M. Bartoszuk, Trans. of the North Ameri. Manuf. Res. Inst. of Sme, XXIX, 343 (2001)

39. P. Niesłony, W. Habrat, P. Laskowski, Mechanik, $8 / 9,71-81(2015)$ 